\title{
Dynamic Product Portfolio Management with Life Cycle Considerations
}

\author{
Ralf W. Seifert ${ }^{\mathrm{a}, \mathrm{b}}$, Jean-Sébastien Tancrez ${ }^{\mathrm{c}, *}$, Işık Biçer ${ }^{\mathrm{a}}$ \\ ${ }^{a}$ Ecole Polytechnique Fédérale de Lausanne, CH-1015 Lausanne, Switzerland \\ bIMD, Chemin de Bellerive 23, P.O. Box 915, CH-1001 Lausanne, Switzerland \\ ${ }^{c}$ Louvain School of Management, Université catholique de Louvain, Chaussée de Binche 151, 7000 Mons, \\ Belgium
}

\begin{abstract}
We consider a portfolio of products in which each product probabilistically transitions through various life cycle stages. The evolution through these life cycle stages is impacted by both marketing support and product launch decisions, which are bound by a financial constraint in the form of restricted working capital. In the model, the joint working capital level serves to finance the portfolio expenditures and is decided a priori by the company. We use an infinite-horizon Markov Decision Process (MDP) to find the optimal decisions to coordinate the operations and finance depending on the composition of the portfolio. We find that the policy optimizing the product launch, marketing support and inventory decisions under a working capital constraint is independent of the correlation between demand levels of the products in the portfolio. We numerically demonstrate that the joint management of the products has a significant positive effect on the total profit, reducing the amount of working capital used per product. We also show that the optimal policy smooths aggregated demand levels as well as cash flows.

Keywords: Product Portfolio Management; Life Cycle; Working Capital; Markov Decision Process; Inventory Control.
\end{abstract}

\section{Introduction}

Companies have to continuously manage their portfolio of product lines. Some prominent examples include the mobile product lines (iPods, iPhones, iPads) managed by Apple Inc. or the product lines of DSLR cameras, such as the Rebel series managed by Canon Inc.

\footnotetext{
${ }^{*}$ Corresponding author. Email: js.tancrez@uclouvain.be; Tel: 003265323538.
} 
For consumer electronics in particular, disregarding the variations in demand across different product life cycle stages may result in high operational costs due to inadequate ordering decisions and revenue shortfalls. In a portfolio of product lines, the product life cycle acquires even more relevance because the upper and lower extremes of demand and revenue may occur simultaneously in multiple product lines and thus be magnified. Therefore, companies may want to smooth the overall levels of demand and revenue by actively managing their portfolio, properly timing product upgrade launches and adequately choosing the amount and timing of marketing support. When firms are financially constrained in making needed investments yet still have to manage multiple products through various life cycle stages, achieving optimal operational and marketing decisions becomes complex. To understand how a company should combine its inventory, product launch and marketing decisions to successfully coordinate its operations and finances, we have brought together three subjects in a unified model: product portfolio management, product life cycle and financial constraints.

We consider the product life cycle as the main source of demand dynamics, which is particularly relevant for products with short life cycles such as high technology goods (Chien et al. (2010)), consumer electronics (Gerhard et al. (2011)), and fashion apparel (Şen (2008)). The product life cycle is represented as a sequence of the following stages: introduction, growth, maturity, decline, and end-of-life (Van der Laan and Salomon (1997); Ahiska and King (2010)). The transient time in these stages is extremely uncertain (stochastic). The firm can use marketing support to increase the probability of a transition to higher demand stages or to increase the resting time at the maturity stage. (The growth and decline stages can span multiple demand levels.) Therefore, within limits, companies can actively manage their product portfolio to achieve more favorable demand realizations. In fact, product life cycle management acquires real meaning at the product portfolio level since harmonizing decisions can be made to smooth aggregated demand and thus income.

Although this overall objective is clear, difficulties arise when the multiplicity of pertinent operational and financial parameters are considered. Products are characterized by varying operational cost parameters, including procurement, holding, and lost sales costs. Moreover, products can have very specific life cycle characteristics, which means each one will be in the market for different lengths of time. Furthermore, demand variability is specific to each product and to its life cycle stage. The complexity of these operational parameters should 
be taken into account when managing a product portfolio. Independent ordering decisions appear inadequate. Instead, companies may achieve higher benefits if they carefully decide when to launch each product and jointly allocate marketing support for each one to smooth total inventory and demand at the portfolio level. This product portfolio management will be particularly important when funding is constrained.

In our study, we include financial constraints in the form of a joint working capital (WC) (Protopappa-Sieke and Seifert (2010)). This WC level refers to a constant level of cash and inventory held by the company (see Section 3 for further discussion). On the one hand, WC that is too tightly constrained may limit the company's ability to purchase adequate levels of inventory to respond to demand peaks. On the other hand, WC that is too loose could increase financial costs. Ernst and Young (2010) found evidence that the top 2000 largest companies in the US and Europe have an aggregate total of up to USD 1.1 trillion in cash (equivalent to $7 \%$ of their sales) unnecessarily tied up in WC. This reference shows that a firm's WC level is an important decision for the management of a product portfolio's operational and financial costs.

In this work, we have devised an infinite-horizon Markov Decision Process (MDP) that includes operational and financial aspects to help manage a portfolio of product lines. With this tool in hand, we examine the following questions:

1. To what extent can active product launch and marketing support decisions improve the management of the product portfolio given homogenous and heterogenous products?

2. How sensitive are the product portfolio revenues (and product launch decisions) to financial constraints in the form of allocated working capital levels?

3. For different working capital levels, what are the most favorable product portfolio compositions?

We find that the policy optimizing the product launch, marketing support and inventory decisions under a working capital constraint is independent of the correlation between demand levels of the products in the portfolio. We numerically demonstrate that the joint management of the products has a significant positive effect on the total profit, reducing the amount of working capital used per product. We also show that the optimal policy smooths aggregated demand levels as well as cash flows.

The remainder of the paper is organized as follows. In Section 2, we review the relevant 
literature. In Section 3, we describe the modeling settings and our mathematical model in detail. In Section 4, we illustrate the use of our tool with numerical examples and discuss managerial implications based on our main observations. In Section 5, we summarize the main results and comment on future research opportunities.

\section{Literature Review}

Our work touches on three important literature streams: product life cycle models, portfolio management, and working capital (WC) constraints for product portfolios.

Within the product life cycle literature, two branches may be distinguished: the first includes the product life cycle for strategic analysis, and the second studies the operational and financial coordination during product rollovers (the time when a product is substituted by an upgrade). In the literature on product life cycle for strategic analysis, Barksdale et al. (1982) explain the Boston Consulting Group's product portfolio matrix and propose to combine it with product life cycle stages to provide a more comprehensive framework for strategic analysis. Similarly, Childerhouse et al. (2002) and Aitken et al. (2003) develop a framework of five parameters (duration of life cycle, time window for delivery, volume, variety and variability) to choose the adequate demand chain for a portfolio of products. With this framework, a UK lighting company restructured its demand chains and as a result reduced lead times and achieved better performance in sales, and customer service. The above studies show the importance of product life cycle in assembling the right product portfolio.

The product rollover literature analyzes the timing of product upgrade launches, as well as the inventory and pricing for the old and upgraded products during these transitions. Billington et al. (1998) conceptually discuss the product rollover problem. They identify that companies may choose a solo-rollover (a single product is in the market) or a dual-rollover (both products may be in the market) depending on the product and market risk of the old and the upgraded products. Wilhelm and Xu (2002) develop a dynamic programming model to decide the technology content of the upgraded product, the optimal pricing and the optimal inventory quantities. Lim and Tang (2006) find the optimal pricing and timing for both solo- and dual-rollover, and show that the choice of strategy depends on the marginal cost of both products. Li et al. (2010) analyze the product rollover problem while considering product substitution, and find that substitution increases profitability during the transition and 
reduces inventory needs for the old product. Most recently, Liao and Seifert (2015) analyze the optimal frequency of new product introductions and analytically prove that the speed of technology diffusion is positively associated with the frequency of the product introductions. This body of literature emphasizes the importance of product life cycle for strategic planning, and highlights the fact that companies need to achieve a balance when they have to manage two coexisting products simultaneously. However, these studies do not consider any interactions between financial and operational decisions. More importantly, they do not address how the interdependence among inventory ordering, marketing support and product launch decisions influence and react to stochastic product life cycle transitions, all of which are crucial, especially in a multi-product setting.

These limitations are partially addressed in quantitative portfolio management models reviewed by Schmidt and Freeland (1992). The following studies are particularly relevant given the scope of our work. Souder (1973) devises a nonlinear, a linear, a zero-one and a profitability index portfolio management model, and then benchmarks it with actual management decisions and outcomes. The author identifies the model that performs best at different stages of the life cycle of the R\&D projects, and highlights the importance of reacting to life cycle dynamics. Fox et al. (1984) focus on project portfolio management while taking into account resource utilization interactions, outcome or technical interactions, and benefit or payoff interactions. They state that considering projects independently can lead to the possibility of "poor selection decisions and resource allocation problems." Also relevant to portfolio dynamics are the theoretical studies by Kleywegt and Papastavrou (1998, 2001) on the time-dependent and stochastic knapsack problem. These two papers analyze when to accept projects given that their rewards and times of arrival are unknown and given that there is a resource constraint for all projects. Their studies highlight the importance of uncertain dynamics on portfolio selection. Most recently, Cooper et al. (1999) make an empirical study of 205 US companies, which are subdivided into four quadrants based on the effectiveness and ease of adoption of their portfolio management method. They find that the most effective companies are those who consistently use financial and strategic methods. From this summary of quantitative models in portfolio management, it is clear that portfolio dynamics and interactions are the most important aspects for the adequate balance of portfolios. Dickinson et al. (2001) develop a non-linear integer optimization model with project interdependencies 
and budgetary and portfolio balance constraints for the project portfolio management of Boeing Company. The authors find that the inclusion of a matrix with values that assess project interdependence helps in "providing balance and supporting the strategy of the enterprise." For further discussion on this literature, we refer to the survey by Kavadias and Chao (2007). Although these models help with portfolio management in static and dynamic settings, they do not examine financially constrained inventory management decisions nor their revenue implications.

Prior research on multi-product inventory management models with WC constraints, i.e. budget and/or capacity constraints, complement the portfolio management literature. In one of the earliest studies, Veinott Jr (1965) creates a general model for a multi-product dynamic inventory problem with partial or complete backlogging, a fixed delivery lag and a budget or storage constraint. Assuming independent demands and no lags in product delivery, the authors find that the base stock policy is optimal. Subsequent studies develop heuristics to approximate the optimal policy for multi-product settings, which include the productioninventory interaction. DeCroix and Arreola-Risa (1998) include a shared constrained resource; Choi et al. (2005) include warehouse capacity; and Ketzenberg et al. (2006) include seasonal demand and constrained production. The authors find how the shared constraint affects the performance of heuristics and under which conditions the joint capacity is under pressure. Lastly, Protopappa-Sieke and Seifert (2011) focus on the interrelation of WC and stocking decisions for functional, innovative and heterogeneous product portfolios. They perform numerical analysis on demand correlation, lead time and payment delays among others. One key finding is that there are "significant risk-pooling benefits in terms of WC requirements under specific demand correlation scenarios." This body of literature demonstrates the strong effects that budget and capacity constraints can have on overall performance and optimal decisions. Nevertheless, much of this analysis does not examine how decisions could be encompassed to address the financial constraint, especially in indeterministic dynamic settings.

The literature on product life cycle, portfolio management, and constrained WC has made important contributions in each specific area, but has not aspired to unify different concepts. Recent works by Ahiska and King (2010) and Hsueh (2011) have combined inventory management for a single product produced through manufacturing or remanufacturing with product life cycle considerations. For each product life cycle stage, Ahiska and King (2010) approx- 
imate the optimal inventory policies with a Markov decision process, and show that these policies are sensitive to set-up costs, changes in demand, and return rates. Hsueh (2011) finds analytical solutions for the lot size, reorder point, and safety stock in each life cycle stage. He finds that the closed-form formulas for the inventory policy are not sensitive to the length of the product life cycle stage. Even though these two papers emphasize the importance of adapting the inventory policy to the product life cycle, their models rest on the single product setting, and overlook the indeterministic nature of the product life cycle by fixing the length of each stage.

Other interdisciplinary studies by Heidenberger et al. (2003), Hess and Lucas (2004) and $\mathrm{Hu}$ and Bidanda (2009) have combined product life cycle considerations and marketing support decisions in a multiple product setting. These studies relate most closely to our work. With the objective of balancing short-term financial stability and long-term competitiveness, Heidenberger et al. (2003) focus on the balance of R\&D investment and marketing support. They argue that too much R\&D investment could unbalance the budget and too little could squeeze future research opportunities. They numerically investigate the changes in the levels of profit for multiple R\&D budgeting rules. However, although the authors develop a complete accounting model, the setting is focused on studying the budgeting of R\&D investments rather than the dynamics of the product portfolio. Hess and Lucas (2004) develop a model for assigning resources to production and marketing research for a portfolio of products, of which only one succeeds. They assume resource-constrained production and that upfront marketing research reduces the uncertainty about which product will succeed. The authors derive analytical solutions and analyze how prior knowledge, marketing research cost, profit margin and production efficiency affect optimality. This work emphasizes the strategic allocation of resources in a product portfolio to achieve adequate production levels and to reduce market uncertainty. However, their findings are limited due to their assumption of a single product success, which precludes the study of the benefits of dynamic portfolio equilibrium in the multi-product case. $\mathrm{Hu}$ and Bidanda (2009) focus on a multi-product setting and examine decision making during the life cycle of a product once it has been launched in a competitive market. The authors state that a Markov decision process is a suitable framework for sequential decision making. The product life cycle is an indeterministic process, but their model lacks more detailed cost accounting. In particular, demand is always fully satisfied on the 
spot, i.e. there is no lead time, no leftover inventory, and no unsatisfied demand.

Based on this review, we can now position our paper and explicitly state its contribution. Our study builds on the product life cycle, portfolio management and WC constrained literature, by joining these three interrelated streams to devise a more complete model for product portfolio management. Since we use a multi-product setting, our model goes beyond the single-product studies by Ahiska and King (2010) and Hsueh (2011). We also go beyond the work by Heidenberger et al. (2003), Hess and Lucas (2004) and Hu and Bidanda (2009) since our detailed accounting explicitly includes three interrelated subjects. In particular, both operations and dynamic decision making are part of our portfolio management model taking the management of WC into account.

\section{Mathematical model}

In this section we devise a mathematical model for a company that jointly manages a portfolio of product lines. For example, the decision maker in our model might be the head of the product portfolio of the DSLR camera lines at Canon Inc. In the portfolio management model, we include the following three key features: 1) there are multiple product lines, where a sequence of product upgrades transition stochastically through their life cycle stages; 2) the portfolio of product lines can be actively managed by deciding the timing of product launches and the levels of marketing support; and 3) the optimal decisions are taken based on expected revenue computation, taking into account inventory and ordering costs. Product launch, marketing support and operational costs are limited by a joint working capital (WC) level (Zeballos, 2013). We use an infinite-horizon Markov Decision Process (MDP) to jointly optimize the operational and financial decisions. In the next subsections, we explain the features of our MDP model in detail. We start by describing the modeling of the product life cycle, first for a single product line. Then, we use the single product line setting as a building block for the multiple product line setting. With the multiple product line setting, we detail the timing of product launches, marketing support, inventory policy and the calculation of rewards. We conclude by commenting on the algorithm to find the optimal decisions for our MDP model. 


\subsection{Product life cycle modeling in a single product line}

As a first step, we model the stochastic nature of the product life cycle for a single product line as a discrete Markov chain (MC). For a given product line, a product stays in the market during the length of its life cycle and is then replaced by a product upgrade. The product life cycle is characterized as a stochastic transition through five sequential stages: introduction, growth, maturity, decline, and end-of-life (Van der Laan and Salomon (1997); Ahiska and King (2010)). These stages capture the differences in the mean and the variance of demand throughout the life of a product. Fig. 1a shows, among many possible scenarios, three possible demand paths for a single product. The x-axis in Fig. 1a represents the time period over the product-life cycle, and the y-axis represents the demand level. In each demand level, we assume that both the mean and the variance of demand remain the same, regardless of the stage in the product life-cycle. For example, both the mean and the variance are the same in the states 1, 1', and 1". Hence, we use the words demand level and mean demand interchangeably. The difference between the demand paths is the growth to higher demand levels and the resting time at a given peak demand level. Naturally, one cannot know in advance how successful a product will be and which of these demand paths will be realized.

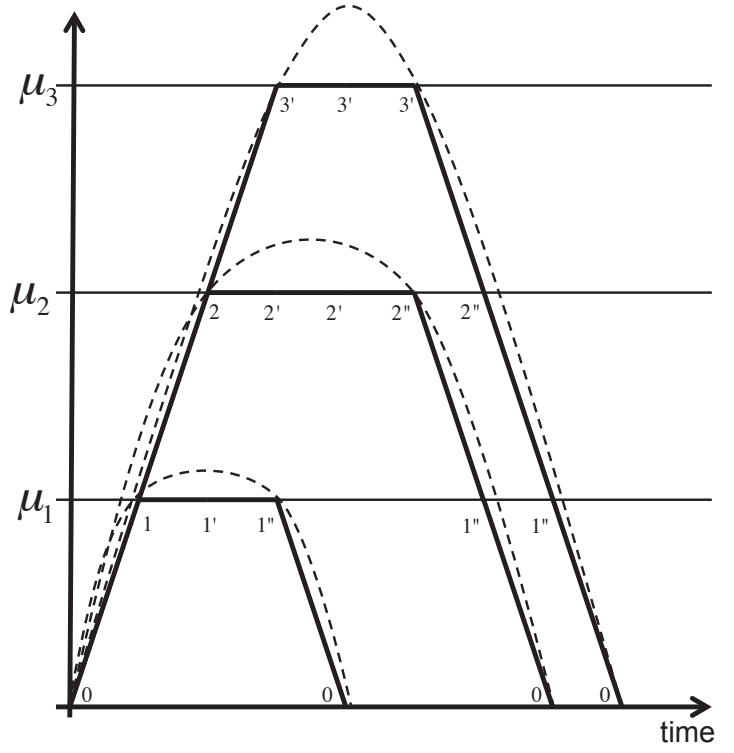

(a)

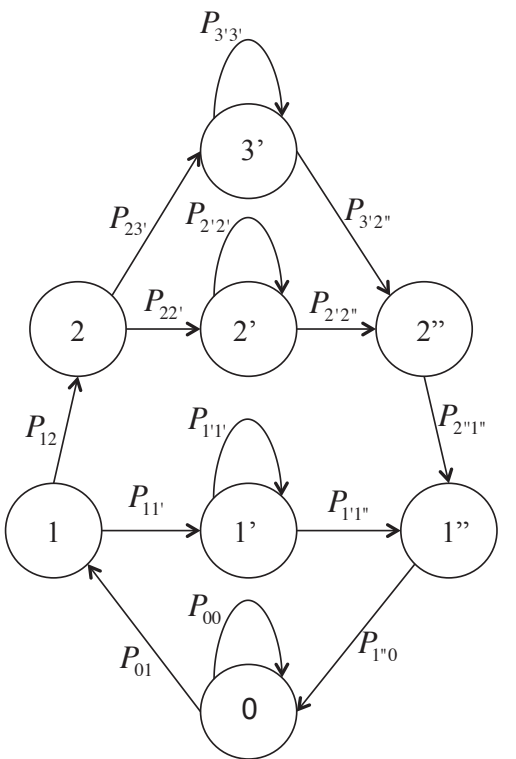

(b)

Figure 1: (a) Potential demand paths for a single product. (b) MC representation of a product line with products that transition through their life cycle stages.

We capture the multiplicity of product life cycle paths and their probability of realization with a MC, as illustrated in Fig. 1b, which shows a MC representation with three different 
demand levels. Each state in the MC lies on one of these demand levels. Product life cycle stages are represented as follows: introduction by the transition $0 \rightarrow 1$; growth by states 1,2 ; maturity by states $1^{\prime}, 2^{\prime}, 3^{\prime}$; decline by states $2^{\prime \prime}, 1^{\prime \prime}$; and end-of-life by the transition $1^{\prime \prime} \rightarrow 0$. Each transition in the MC is assigned a probability that determines the likelihood for each demand path. Note that, in this model, the demand variance may also depend on the life cycle stage. Typically, the variance could be higher in the early stages of the life cycle and decrease progressively. We acknowledge that this MC excludes the possibility of multiple ups and downs of demand levels, but this is in inline with the classical product life cycle representation (Day (1981)).

In Fig. 1b, when the MC state is 0 , there is no demand, and there is no product in the market. This is the case until there is a transition to state 1 , which means that a product upgrade has been launched. Therefore, the MC models a product line in which a product transitions through its life cycle stages, followed by a period of no products in the market, until a product upgrade is launched and so on. In our model, a company can decide to either launch a product upgrade or postpone the launch. Moreover, it can use marketing support to increase the probabilities of reaching higher demand levels for a given product. These two types of decisions affect the transition probabilities in different ways:

- Product launch decision: if a product line does not have a product on the market (state 0), a company can decide to postpone launching an upgrade and pay nothing, or it can decide to bear the investment cost and launch an upgrade. If the product launch is postponed, the transition $0 \rightarrow 0$ is assigned a probability of 1 , and if the product upgrade is launched the transition $0 \rightarrow 1$ is assigned a probability of 1 . For example, in Fig. 4, at period 0 , the company launches the upgrade for product line 1, and postpones the launch of product line 2 for two periods from $t_{1}$ to $t_{2}$.

- Marketing support decision: if a product is in the growth states (states 1,2 in Fig. 1b), the company may use higher levels of marketing support to increase the probability of reaching higher demand levels. With higher marketing support, the transition probabilities to reach a higher demand level (e.g. $P_{12}$, and $P_{23^{\prime}}$ in Fig. $1 \mathrm{~b}$ ) will increase. If a product is in the maturity states $\left(1^{\prime}, 2^{\prime}, 3^{\prime}\right.$ in Fig. 1b), the company may use higher levels of marketing support to increase the probability of longer resting times in the maturity stage (e.g. $P_{1^{\prime} 1^{\prime}}, P_{2^{\prime} 2^{\prime}}$ and $P_{3^{\prime} 3^{\prime}}$ in Fig. 1b). 
We use $\mathcal{J}$ to denote the states of the MC. Thus,

$$
\mathcal{J}=\left\{0,1,2,1^{\prime}, 2^{\prime}, 3^{\prime}, 1^{\prime \prime}, 2^{\prime \prime}\right\}
$$

Let $\mathcal{A}$ denote the set of all possible actions:

$$
\mathcal{A}=\left\{a_{0}, a_{1}, a_{2}\right\}
$$

where $a_{0}$ is the postponement of the investment, $a_{1}$ is the product launch, and $a_{2}$ is the marketing support. We use $\mathcal{A}_{j}$ to denote the set of possible actions in state $j \in \mathcal{J}$. Then,

$$
\mathcal{A}_{j}= \begin{cases}\left\{a_{0}, a_{1}\right\} & \text { if } j \in\{0\} \\ \left\{a_{0}, a_{2}\right\} & \text { if } j \in\left\{1,2,1^{\prime}, 2^{\prime}, 3^{\prime}\right\} \\ \emptyset & \text { if } j \in\left\{2^{\prime \prime}, 3^{\prime \prime}\right\}\end{cases}
$$

The set of feasible pairs of states and actions $\mathcal{L}$ is

$$
\begin{aligned}
\mathcal{L}=\left\{\left(0, a_{0}\right),\left(0, a_{1}\right),\left(1, a_{0}\right),\left(1, a_{2}\right),\left(2, a_{0}\right),\left(2, a_{2}\right),\left(1^{\prime}, a_{0}\right),\right. \\
\left.\left(1^{\prime}, a_{2}\right),\left(2^{\prime}, a_{0}\right),\left(2^{\prime}, a_{2}\right),\left(3^{\prime}, a_{0}\right),\left(3^{\prime}, a_{2}\right),\left(1^{\prime \prime}, \emptyset\right),\left(2^{\prime \prime}, \emptyset\right)\right\} .
\end{aligned}
$$

The formulation of the set of the feasible pairs allows us to characterize the investment options in each state. In the following, we analyze the inventory model to formulate the reward function and then give the expected value function for the optimal solution.

\subsection{Rewards in the MDP}

We now consider a single product and analyze the dynamics of the inventory model to calculate the operational profit in each state. The operational profit is defined as the income from sales minus procurement costs, holding costs and loss of goodwill. We use $D_{j}$ to denote the demand for a product in state $j \in \mathcal{J}, S_{j}$ the target order-up-to level, $W_{j}$ the actual order-up-to level, $p$ the selling price per unit, $c$ the cost of product per unit, $h$ the holding cost per unit per period, $g$ the goodwill cost per unit, and $K$ the available working capital at the beginning. We assume that excess demand is lost. This assumption is relevant for the products with substitutes available in the market (Nagarajan and Rajagopalan, 2008) 
and also for the niche products whose demand is positively correlated with their availability in the market (Anderson, 2009; Biçer, 2015). Nevertheless, the lost-sales assumption is not restrictive in our setting because our model can be easily adapted for analyzing a backorder model as will be discussed below. The cost of underage is the sum of the cost of lost sales and the goodwill cost (i.e., $(p-c+g))$. The replenishment lead time is equal to one period. Thus, the orders placed with the supplier are replenished in the next period. The target order-up-to level might change as the MDP moves from one state to another. If the target order-up-to level is lower than the available inventory on hand at the end of the previous state, the actual order-up-to value becomes more than the target. Therefore, we have the following recursive relationship:

$$
W_{j}=\max \left\{S_{j}, W_{j-1}-D_{j-1}\right\}, \quad \forall j \in \mathcal{J},
$$

where $(j-1) \in \mathcal{J}$ denotes the previous state. The inventory model can be illustrated as in Figure 2. We simplify the lost sales model in Figure 2 by reducing it to the form of a

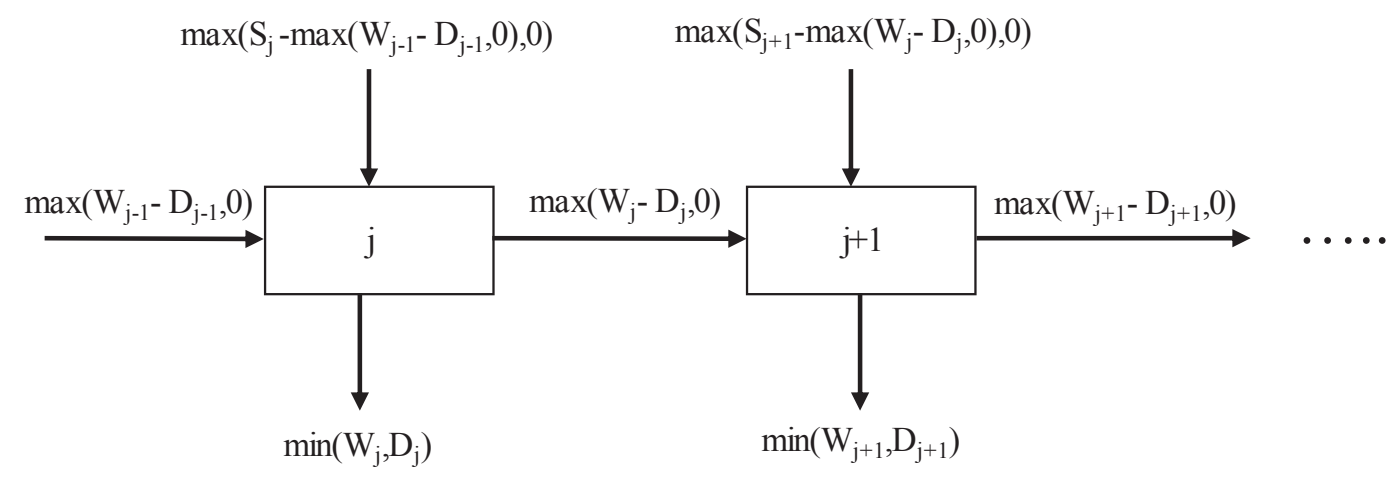

Figure 2: Lost-Sales Model

backorder model. We define the backordering cost of the equivalent backorder model as the underage cost of the lost-sales model, which is equal to $(p-c+g)$. Then, the lost-sales model can be simplified as illustrated in Figure 3. 


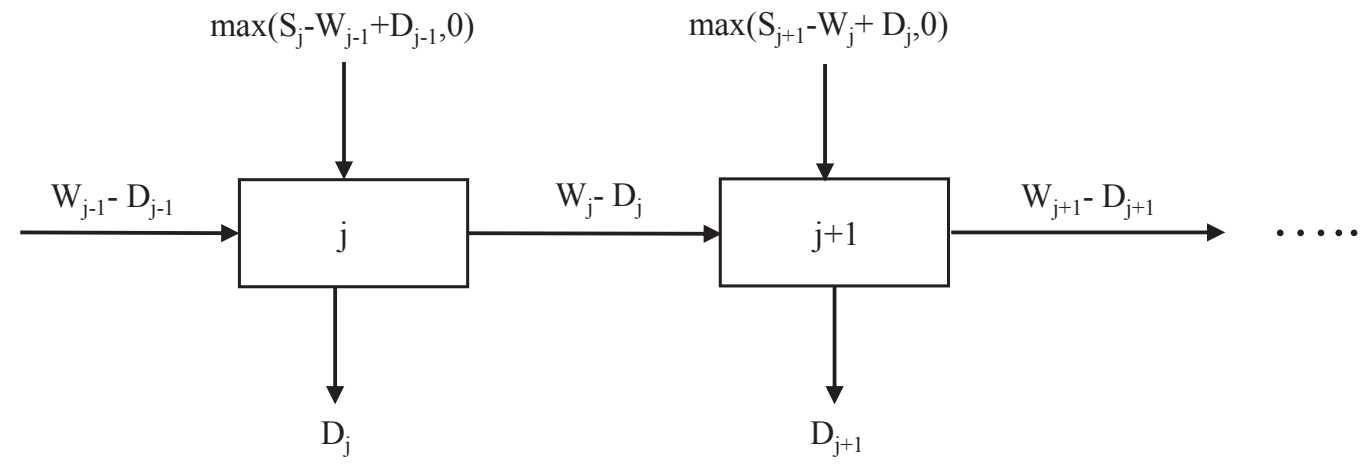

Figure 3: The Equivalent Backorder Model

The expected profit in state $j+1$ forecasted in state $j$ is formulated as follows:

Max: $\quad \mathrm{E}\left[\Pi_{j}\left(S_{j+1}\right)\right]=\mathrm{E}\left(p D_{j}-h \max \left(W_{j}-D_{j}, 0\right)-(g+(p-c)) \max \left(D_{j}-W_{j}, 0\right)\right.$

$$
\left.-c \max \left(W_{j+1}-S_{j}+D_{j}, 0\right)\right)
$$

subject to

$$
c \max \left(W_{j+1}-S_{j}+D_{j}, 0\right) \leq K-A_{j}
$$

where $A_{j}$ is the cost of any action taken in state $j$ for the product. We note that the actual order-up-to level converges to the target value (i.e., $W_{j} \rightarrow S_{j}$ ) in long run. Then, the optimal order-up-to level conditional on the working capital level $K$ is approximated by

$$
S_{j+1}^{*} \mid K=\min \left(F_{j}^{-1}(\beta), \frac{K-A_{j}}{c\left(S_{j+1}-S_{j}+D_{j}\right)^{+}} F_{j}^{-1}(\beta)\right)
$$

where $F_{j}^{-1}($.$) denotes the inverse of F_{j}($.$) , which is the conditional distribution of demand D_{j+1}$ forecasted in state $j$, and $\beta$ is the critical fractile:

$$
\beta=\frac{g+(p-c)}{h+g+(p-c)}
$$

We acknowledge the simplicity of this order adjustment policy, but believe it to be sufficient given the focus and the scope of our paper. Indeed, it may help with the clarity of the subsequent analysis. Further work could examine more sophisticated order adjustment policies, for example building on the studies of McGavin et al. (1997) and Moon and Silver (2000). 
We assume that the target working capital level is equal to $K$; therefore, any cash flow to keep the working capital at the target level is included in the reward function. If the available working capital is less than this amount, the company issues short-term debt with an interest rate of $\theta$. This is a standard assumption in the related literature (Hu et al., 2010; Li et al., 2013; Biçer and Seifert, 2015). Li et al. (2013) argued that this assumption is also consistent with the working mechanisms of companies. When the working capital in a company is less than the target level, equity holders may be required to subscribe additional capital to bring the working capital to its target value. If the working capital exceeds the target level, the company pays out dividends to equity holders (Li et al., 2013; Biçer and Seifert, 2015). Then, the reward function in state $j$ given the working capital $K$ is formulated as:

$$
R(j \mid K)= \begin{cases}\Pi_{j}\left(S_{j+1}^{*} \mid K\right)-A_{j}, & \text { if } \Pi_{j}\left(S_{j+1}^{*} \mid K\right)-A_{j}>0 \\ (1+\theta)\left(\Pi_{j}\left(S_{j+1}^{*} \mid K\right)-A_{j}\right), & \text { otherwise. }\end{cases}
$$

The expected value for the optimal solution starting from the state $j$ is

$$
V(j \mid K)=\max _{a \in \mathcal{A}} \mathrm{E}\left\{R(j \mid K)+\gamma \sum_{j^{\prime} \in \mathcal{J}} P\left(j^{\prime} \mid j, a\right) V\left(j^{\prime} \mid K\right)\right\}
$$

where $a \in \mathcal{A}$ is the action taken in state $j, \gamma$ is the discount factor, and $P\left(j^{\prime} \mid j, a\right)$ is the transition probability from state $j$ to $j^{\prime}$ given that the action $a$ is taken. Then, the optimal set of actions is found by the value iteration method.

\subsection{Multiple product lines}

To model the product life cycle for multiple product lines, we extend the single product line formulation presented in the previous section. In the multiple product line representation, $M$ products may be at different stages of their product life cycle, and their demand paths may have different maximum levels and different realization probabilities. We follow standard practice and include all these features into an aggregated Markov chain ( $\mathrm{Hu}$ and Bidanda (2009)).

The aggregated MC for multiple product lines is defined as follows: The states of the MC are composed of life cycle states for each individual product, i.e. their respective demand level. For example, if a portfolio of two product lines is in state $\left\{1,2^{\prime}\right\}$, it means that Product 1 is in the growth state 1 , and Product 2 is in maturity state $2^{\prime}$. Transition probabilities 


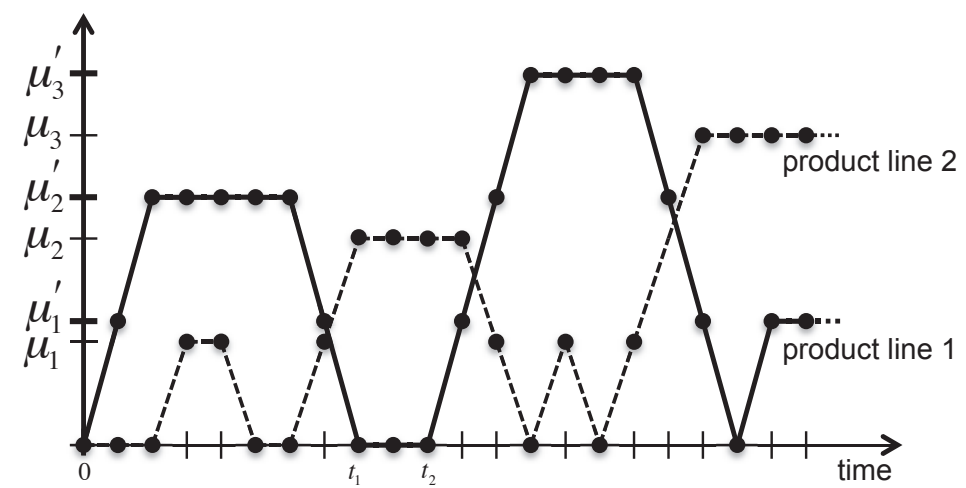

Figure 4: Example of the evolution of two product lines, where products transition through life cycle stages and have different demand levels over time.

for the overall MC are calculated by multiplying single product line state transitions. With this aggregated MC representation, the products in the product lines can be at different life cycle stages simultaneously. Their demand paths may have different realization probabilities depending on how the individual transition probabilities are adjusted. Moreover, each product may have different intermediate and maximum demand levels. Fig. 4 shows an instance of the evolution of two product lines from the $\mathrm{MC}$ model over time. For every product line, the timing of product upgrade launches and marketing support can be actively managed to influence the product life cycle evolution.

We now extend our analytical results, given in Section 3.2, to a multi-product problem. We use the superscript $m \in\{1,2, \ldots, M\}$ to denote the product types and the subscript $j \in \mathcal{J}$ to denote the states. Let $D_{j}^{m}$ be a random variable denoting the demand for product $m$ in state $j, S_{j}^{m}$ the target order-up-to level, $W_{j}^{m}$ the actual order-up-to level, $p^{m}$ the retail price per unit, $c^{m}$ the cost of product per unit, $h^{m}$ the holding cost per unit per period, $g^{m}$ the goodwill cost per unit, and $K$ the total working capital level available at the beginning. We denote by $j_{i}^{m}$ the state of product $m$ in period $i$. Thus, the demand of the product in state $j_{i+1}^{m}$ is denoted by $D_{j+1}^{m}$, for example. We also use $J_{i}^{M}$ to denote a column vector with $M$ elements that gives the states of the products in period $i$ :

$$
\boldsymbol{J}_{i}^{M}=\left[\begin{array}{c}
j_{i}^{1} \\
j_{i}^{2} \\
\vdots \\
j_{i}^{M}
\end{array}\right]
$$


The mathematical model (3.6)-(3.7) is written for the multi-product model as follows:

$$
\begin{aligned}
& \text { Max: } \quad \mathrm{E}\left[\sum_{m=1}^{M} \Pi_{j}^{m}\left(S_{j+1}^{m}\right)\right]=\mathrm{E}\left[\sum _ { m = 1 } ^ { M } \left(p^{m} D_{j}^{m}-h^{m} \max \left(W_{j}^{m}-D_{j}^{m}, 0\right)\right.\right. \\
& -\left(g^{m}+\left(p^{m}-c^{m}\right)\right) \max \left(D_{j}^{m}-W_{j}^{m}, 0\right) \\
& \left.\left.-c^{m} \max \left(W_{j+1}^{m}-S_{j}^{m}+D_{j}^{m}, 0\right)\right)\right],
\end{aligned}
$$

subject to

$$
\sum_{m=1}^{M} c^{m} \max \left(W_{j+1}^{m}-S_{j}^{m}+D_{j}^{m}, 0\right) \leq K-\sum_{m=1}^{M} A_{j}^{m},
$$

where $A_{j}^{m}$ is the cost of action taken for the product $m$. In Equation 3.14, we constrain that the total cost of marketing and production investments cannot exceed the available working capital. Thus, the optimal order-up-to level for each product conditional on the working capital level $K$ is approximated by

$$
S_{j+1}^{m^{*}} \mid K=\min \left(F_{j}^{-1}\left(\beta_{m} \mid a_{j}^{m}, m\right), \frac{K-\sum_{m=1}^{M} A_{j}^{m}}{\sum_{m=1}^{M} c^{m}\left(S_{j+1}^{m}-S_{j}^{m}+D_{j}^{m}\right)^{+}} F_{j}^{-1}\left(\beta_{m} \mid a_{j}^{m}, m\right)\right),
$$

where $F_{j}^{-1}\left(\cdot \mid a_{j}^{m}, m\right)$ denotes the inverse of $F_{j}\left(\cdot \mid a_{j}^{m}, m\right)$, which is the conditional distribution of demand $D_{j+1}^{m}$ for product $m$ forecasted in state $j_{i}^{m}, a_{j}^{m} \in \mathcal{A}$ is the action taken for the product $m$, and $\beta_{m}$ is the critical fractile:

$$
\beta^{m}=\frac{g^{m}+\left(p^{m}-c^{m}\right)}{h^{m}+g^{m}+\left(p^{m}-c^{m}\right)}
$$

Then, the reward function in period $i$ is formulated as follows:

$$
R\left(J_{i}^{M} \mid K\right)= \begin{cases}\sum_{m=1}^{M}\left[\Pi_{j}^{m}\left(S_{j+1}^{m}\right)-A_{j}^{m}\right], & \text { if } \sum_{m=1}^{M}\left[\Pi_{j}^{m}\left(S_{j+1}^{m}\right)-A_{j}^{m}\right]>0 \\ (1+\theta) \sum_{m=1}^{M}\left[\Pi_{j}^{m}\left(S_{j+1}^{m}\right)-A_{j}^{m}\right], & \text { otherwise. }\end{cases}
$$

The expected value for the optimal solution starting from the state $J_{i}^{M}$ is

$$
V\left(J_{i}^{M} \mid K\right)=\max _{a_{i}^{M} \in \mathcal{A}^{M}} \mathrm{E}\left\{R\left(J_{i}^{M} \mid K\right)+\gamma \sum_{J_{i+1}^{M} \in \mathcal{J}^{M}} P\left(J_{i+1}^{M} \mid J_{i}^{M}, a_{i}^{M}\right) V\left(J_{i+1}^{M} \mid K\right)\right\}
$$


where $a_{i}^{M} \in \mathcal{A}^{M}$ is the set of possible actions for all the products in period $i, \gamma$ is the discount factor, and $P\left(J_{i+1}^{M} \mid J_{i}^{M}, a_{i}^{M}\right)$ is the transition probability from $J_{i}^{M}$ to $J_{i+1}^{M}$ given that the actions $a_{i}^{M}$ are taken.

Using Equation (3.15), we develop an allocation policy for the total working capital among different products.

Lemma 1. The amount of capital allocated to product $m$ in period $i$ to cover the production and marketing expenses of the product is estimated by

$$
K_{i}^{m}=\frac{K}{\sum_{m=1}^{M} c^{m}\left(S_{j+1}^{m}-S_{j}^{m}+D_{j}^{m}\right)^{+}} c^{m}\left(S_{j+1}^{m}-S_{j}^{m}+D_{j}^{m}\right)^{+}
$$

We now develop the following theorem to simplify the calculation of the value function in the multi-product case.

Theorem 1. Equation (3.18) can be written as the sum of Equation (3.11) as follows:

$$
V\left(J_{i}^{M} \mid K\right)=\sum_{j_{i}^{m} \in J_{i}^{M}} V\left(j_{i}^{m} \mid K_{i}^{m}\right)
$$

In Appendix A, we give the proof of the theorem. We formulate the problem as a linear programming model. We then use dual variables to show that total profit for a multi-product problem depends on the marginal demand distributions of each product, not on the multivariate demand distribution. Therefore, the value function for the vector $J_{i}^{M}$ is independent from the correlation between the demand for the products in the product portfolio. Then, the optimal set of actions is found by the value iteration method.

To conclude this section, we briefly comment on how the optimal decisions are found. An optimal policy is a set of unique decisions associated with each state of the system. To find the optimal decision we use the standard iterative method (see Puterman (1994)). The algorithm has been implemented using Matlab. The computation time for each iteration grows at a rate of $O\left(|\mathcal{J}|^{M \times 2}|\mathcal{A}|\right)$, where $|\mathcal{J}|$ and $|\mathcal{A}|$ are the sizes of state and action vectors for a single product, respectively. The total computation time is polynomial for a finite number of products if a stopping rule based on a fixed threshold value is determined ex ante (Littman et al., 1995). The size of the state vector (i.e., $|\mathcal{J}|^{M}$ ) depends on both the number of products and the 
number of life-cycle stages. It increases exponentially as the number of products increases. In our model, the number of life-cycle stages is fixed. Thus, the total computation time only depends on the number of products, increasing exponentially in the number of products. The time needed to solve one instance for different portfolio sizes is the following: $0.14[\mathrm{~s}]$ for $M=1,0.23[\mathrm{~s}]$ for $M=2,0.60[\mathrm{~s}]$ for $M=3$, and $145[\mathrm{~s}]$ for $M=4$.

\section{Numerical Analysis}

In this section, we illustrate the use of our product portfolio management tool while answering our main research questions (see Section 1).

\subsection{Product and system parameters}

For our numerical studies, we use as a foundation the classification of product types into functional and innovative products proposed by Fisher (1997). These two product types, by definition, have opposing operational specifications - low vs high profit margin, low vs high uncertainty - and consequently different financial needs, which allows us to illustrate the use of our model well. In Table 1, we summarize the operational parameters for the functional and innovative products used for subsequent illustration. These values are within the range specified by Fisher (1997). In addition, we use the following financial parameters: a longterm annual interest rate of $r=8 \%$, and a short-term debt premium of $\theta=24 \%$. Then, the discount factor calculated as $\gamma=1 /(1+r) \approx 0.93$.

\begin{tabular}{l|rr} 
Description & Functional & Innovative \\
\hline Procurement cost & 1.00 & 1.60 \\
Selling price & 1.10 & 2.32 \\
Profit margin & $10 \%$ & $45 \%$ \\
Holding cost & 0.15 & 0.50 \\
Loss of goodwill & 0.01 & 0.35 \\
Coefficient of variation & 0.15 & 0.50 \\
Low mean demand & 100 & 100 \\
High mean demand & 150 & 300
\end{tabular}

Table 1: Operational parameters for functional and innovative products.

In Table 1, we specify a low and a high mean demand level. In our numerical experiments, we then increase the mean demand at equal intervals between these two demand levels. To illustrate our results, we apply a lognormal distribution to represent demand uncertainty. 
Here, the standard deviation is calculated using a given mean demand level and the coefficient of variation for a particular product type. For example in Fig. 1b, the demand for functional products has the following pairs of mean and standard deviation: $(100,15)$ for $\left\{1,1^{\prime}, 1^{\prime \prime}\right\}$, $(125,18.8)$ for $\left\{2,2^{\prime}, 2^{\prime \prime}\right\}$, and $(150,22.5)$ for $\left\{3^{\prime}\right\}$. Likewise, the demand for innovative products has the following pairs: $(100,50)$ for $\left\{1,1^{\prime}, 1^{\prime \prime}\right\},(200,100)$ for $\left\{2,2^{\prime}, 2^{\prime \prime}\right\}$, and $(300,150)$ for $\left\{3^{\prime}\right\}$. For other values of demand levels, the parameters will be determined in a similar manner. The model can handle general demand distributions (i.e., this setting is for illustrative purposes only).

In our MDP model, we differentiate two key decisions: product launch and marketing support. At state 0, we either decide to "launch" a product upgrade, or to "postpone" it. The estimated benefit from a product launch is defined as the profit margin times the product's low mean demand times the profit margin. The corresponding cost of a product launch is kept proportional to the estimated benefits. In our experiments, we used $10 \%$ for both types of products. Postponing the product has no benefits and no cost. At the growth, maturity and decline states, we limit the actions to two: "no marketing support" and "active marketing support." No marketing support sets the probability of the transitions from the growth states to a higher state and from the maturity states to themselves equal to a default value of 0.3 and with zero cost. Active marketing support sets the probability of those transitions equal to 0.8. The estimated marginal benefit of active marketing support is defined as the change from the default probability times the current state's mean demand times the profit margin. The corresponding cost is proportional to the estimated marginal benefits. In our experiments, it is also assumed to be $10 \%$ for both types of products.

The above parameter setting serves to illustrate the use of our product portfolio management tool and to demonstrate the types of managerial insights that can be fostered by addressing our main research questions. The parameters can be readily adjusted and a similar analysis can be carried out.

\subsection{Single product WC management}

The objective of this section is to understand how WC affects the reward of single-product upgrades, where each product stochastically transitions through its various life cycle stages. For this, we use the average reward per WC as a metric since it measures the effectiveness of the use of WC and can be used to compare portfolios with different sizes and compositions. 
Fig. 5 shows the change in average reward per WC for different WC levels for single product lines of functional and innovative products. For both types of products, the effects of WC can be divided into three regions: In Region I, the company does not launch any products because the $\mathrm{WC}$ is too restricted to sustain profitable operations. In particular, insufficient inventory will result in unsatisfied demand, low revenues and high loss-of-goodwill costs. Since shortterm debt costs are too high, launching a product becomes prohibitively expensive. With no product in the market, we still incur the financial cost of holding capital, which results in a negative objective function value. In Region II, the WC level is high enough to obtain positive rewards. This is because the company can order enough inventory to satisfy demand and increase its revenues to pay for the product launch and marketing support. In fact, as WC increases, the average reward per WC increases. This trend continues until the optimal WC level is reached. The optimal WC level corresponds to the point with the highest average reward per WC. At this point, the company dedicates the ideal level of WC to efficiently run its operations without incurring unnecessary financial costs. In Region III, further increases in WC only yield deminishing returns. The financial costs of holding such high levels of WC cannot be compensated by the benefits from higher ordering given limited demand potential.

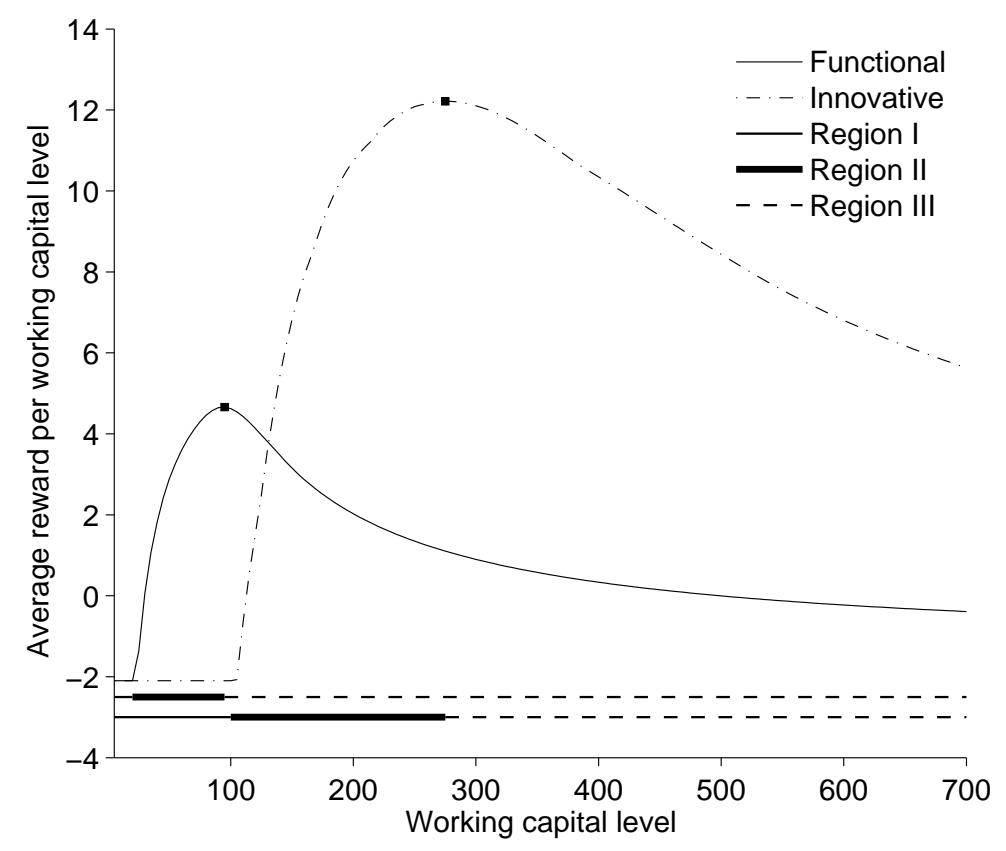

Figure 5: WC efficiency for a single functional and innovative product. $(\mathrm{M}=1)$

The MDP model at hand can help categorize these three regions for a given product and find the optimal WC levels. It also makes it possible to examine the difference in WC 
requirements between a functional and an innovative product (Fig. 5). The functional product has a lower launching and optimal WC level than the innovative product, and it has a lower average reward per WC level than the innovative product. Subsequently, we always determine and use the $\mathrm{WC}$ level where the average reward per $\mathrm{WC}$ is maximized.

Our results in this part can be interpreted as follows. Innovative products have higher demand uncertainty and higher profit margins than standard products. Optimal inventory levels are higher for the innovative products, suggested by the critical-fractile solution (i.e., Equation (3.9)). Therefore, innovative products require higher WC levels than standard products. The average reward per WC level is also higher for the innovative products than standard products due to their higher profit margins.

\subsection{WC benefits for multiple products}

To examine the multi-product case, we initially choose homogenous product portfolios, meaning that products are of identical type: either all functional or all innovative. With these specifications, we analyze the impact of the size of the portfolio on the required WC per product. In Fig. 6(a), we illustrate the optimal WC per product for product portfolio sizes varying from 1 to 4 products. As the size of the product portfolio increases, the required WC per product diminishes because of the opportunities to better time the financial support to reduce the portfolio's overall financial requirements. We will explore this further in Section 4.4. In Fig. 6(b), we see the optimal average reward per WC for different product portfolio sizes. Reward per WC clearly improves with an increased number of products. Therefore, a joint WC management for the whole product portfolio will use resources more effectively, dynamically allocating the resources among the product lines, while increasing the system reward. To understand the forces that cause this improvement, we analyze the product launch and marketing decisions in more detail in the next subsections.

\subsection{Optimal decisions for dynamic portfolio management}

Next, we analyze how launching and marketing decisions help the dynamic management of the product portfolio.

\subsubsection{Benefits of optimal decision making}

For clarity of analysis and presentation, we first focus on a homogenous product portfolio of two similarly innovative products. In Fig. 7(a), we present the optimal decisions for all states. 

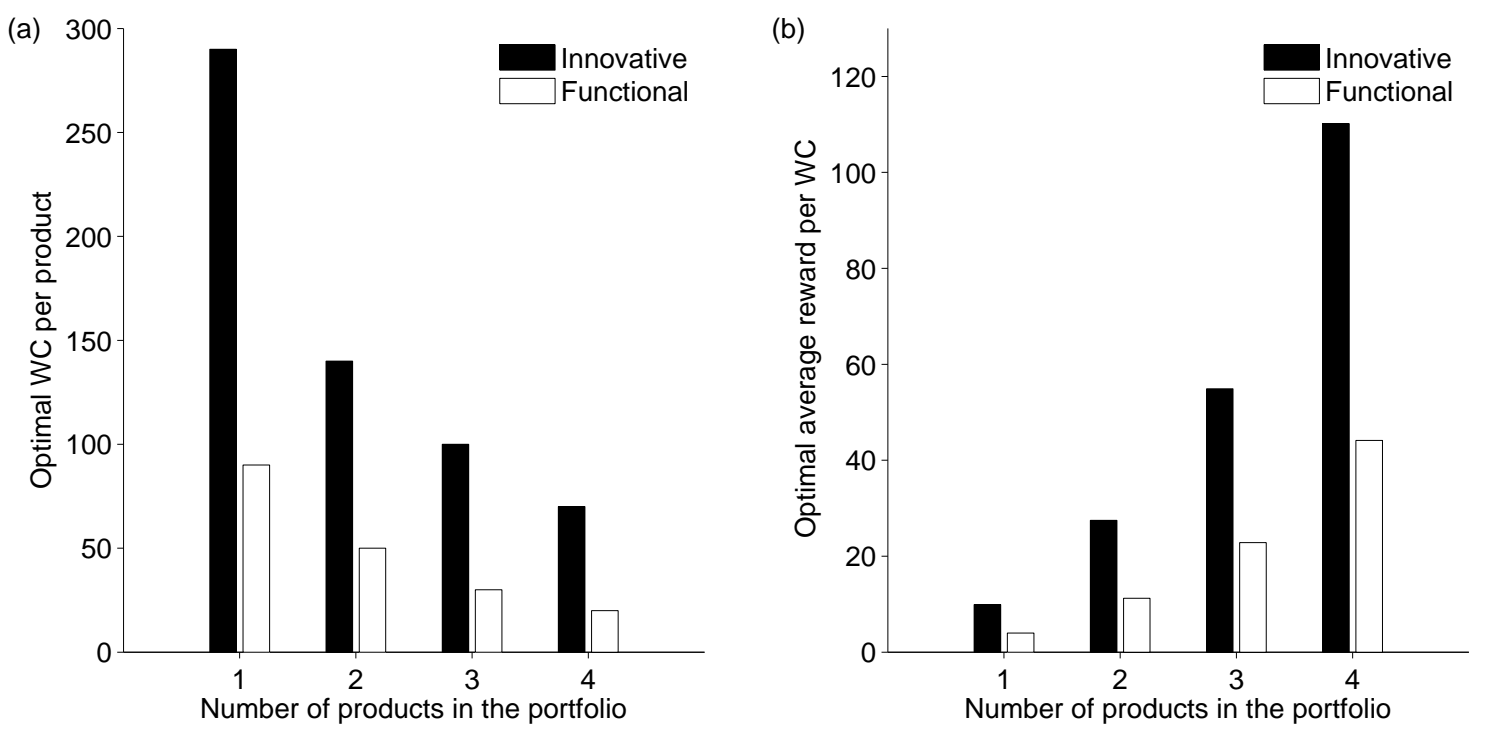

Figure 6: (a) Optimal WC per product for different portfolio sizes. (b) WC efficiency metric for different portfolio sizes. $(\mathrm{M}=1 \ldots 4)$
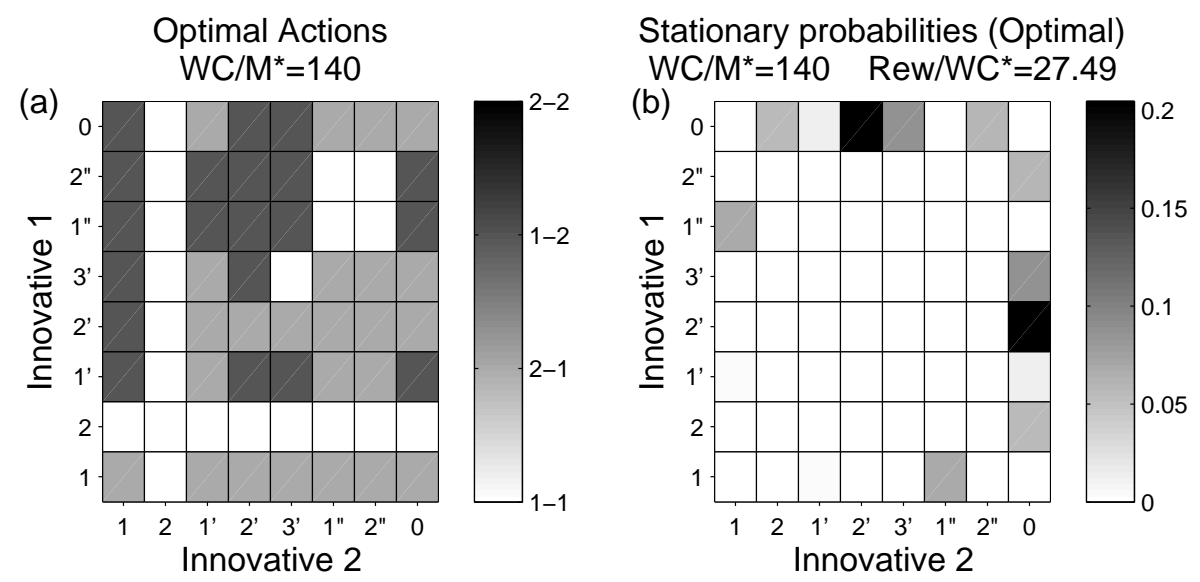

Figure 7: (a) Optimal decisions at the optimal WC level. (b) Stationary probabilities from the optimal decisions. (Homogenous portfolio of innovative products, $\mathrm{M}=2$ )

Three different colors represent different combinations of individual decisions, labeled " 1 " for no investment and "2" for active investment. For instance, the grey squares in Fig. 7(a), labeled "1-2" or "2-1", mean that the company provides marketing support to one product but not the other. In Fig. 7(b), the stationary probabilities of all states that derive from the optimal decisions are presented. The darker the square, the higher the probability that the system is in the corresponding state. The states with the higher probabilities are those in which one product is in $2^{\prime}$ while the other is in 0 . This means that the timing is deliberately set to ensure so that one product is at a high level of demand while the other is at its lowest. This reduces the likelihood of periods with high expenditures or high demand levels since 
these cases require more resources. Fig. 7(a) shows that the equilibrium is achieved through carefully timed decisions. Symmetric states with respect to the main diagonal have symmetric decisions, as expected, since both products have the same characteristics. For example, the decisions in state $\left\{3^{\prime}, 0\right\}$ are equivalent to those in $\left\{0,3^{\prime}\right\}$. In these states, marketing support is provided to the successful product to keep it at its top demand level. In the main diagonal, asymmetric decisions may be used to "break the tie" when both products are in the same state. For example, in state $\{0,0\}$, which is highly improbable, only one product is launched while the launch of the other product is postponed.

To put our results into context, we establish two benchmark strategies: (a) "Cautious" and (b) "Greedy". A Cautious strategy means never investing in marketing and always launching a product when in state 0 . In Fig. 8(a), the stationary probabilities for this strategy show that products are seldom in the top state while they are often simultaneously in the same states. As a result, the reward per WC with this strategy is negative and much lower than the optimal (-0.32 vs. 27.5*). A Greedy strategy means always investing in marketing and
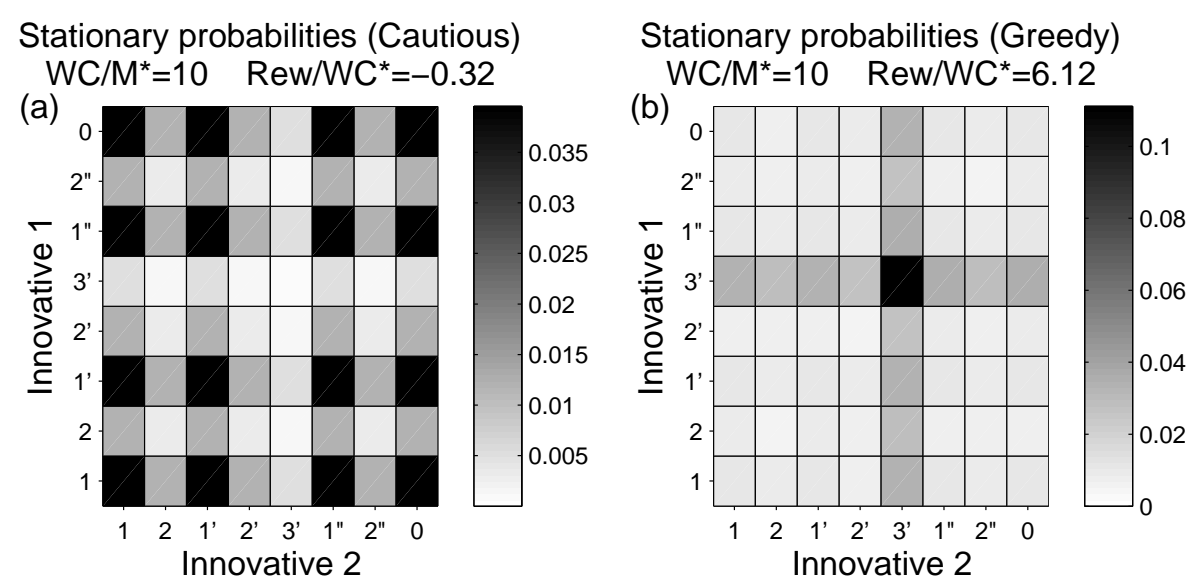

Figure 8: Stationary probabilities for the (a) Cautious and (b) Greedy strategies. (Homogenous portfolio of innovative products, $\mathrm{M}=2$ )

always launching a product. In Fig. 8(b), the stationary probabilities for this strategy show that products are often at their top demand level. Although this result might seem desirable, having both products at the top level puts a great deal of pressure on the inventory ordering capacity. This results in a relatively low reward, much lower than the one from the optimal decisions computed with the proposed portfolio management tool (6.12 vs. 27.5*). In reality, the optimal policy might be hard to define from intuition. For example, from the optimal decisions in Fig. $7(\mathrm{a})$, we see that in state $\{2,2\}$, it is optimal not to invest, while in state 
$\left\{2^{\prime}, 2^{\prime}\right\}$ it is optimal to invest in only one product. Therefore, optimal decisions may not follow intuition, even in simple settings, and alternative policies might be far from the optimal reward for a company.

4.4.2. Reaching stable aggregate demand levels
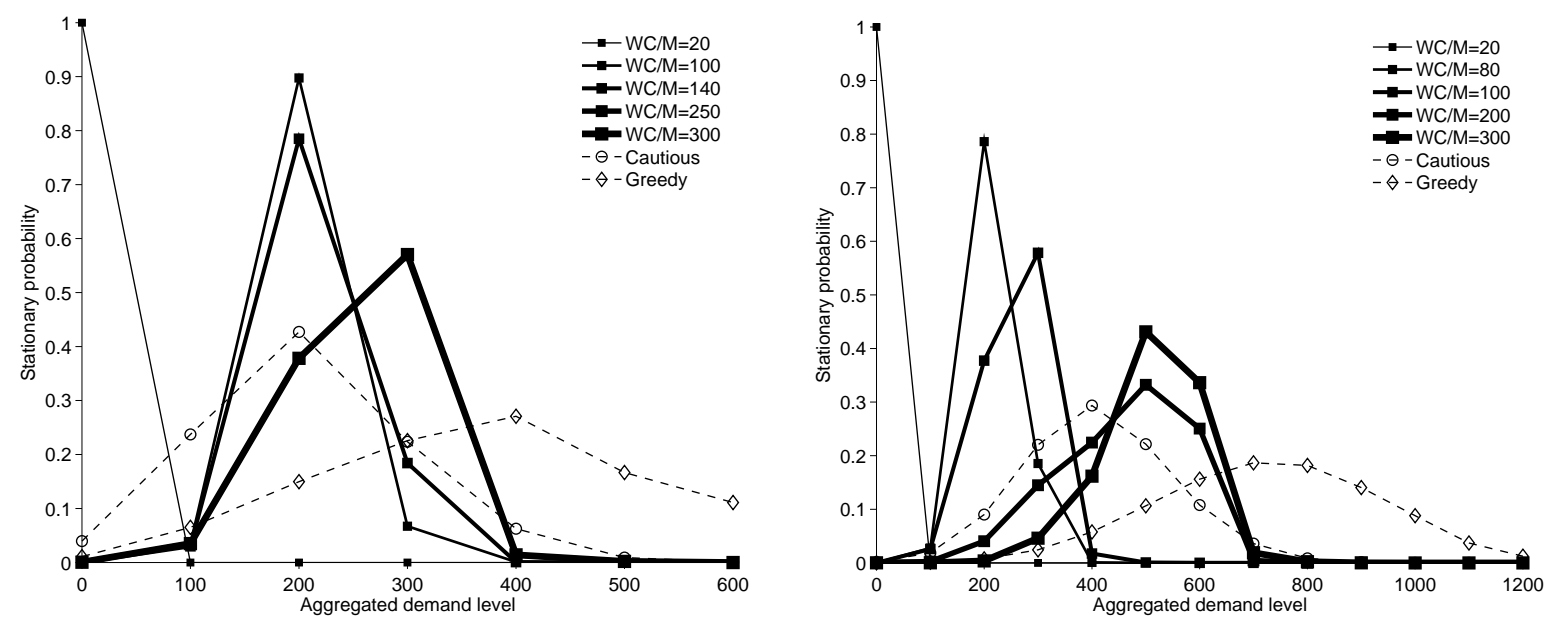

Figure 9: Aggregated demand levels with their respective stationary probabilities for different levels of WC and the Cautious and Greedy policies. (a) $\mathrm{M}=2$ (b) $\mathrm{M}=4$. Both portfolios have similar innovative products.

Next, we analyze the stationary probabilities of the aggregated demand for homogenous portfolios of 2 and 4 innovative products when using the optimal decisions (Fig. 9). In both Fig. 9(a) and 9(b), we compare the stationary probabilities of the aggregated demand for different WC levels and for the Cautious and Greedy strategies. Firstly, it can be seen that the optimal decisions smooth the aggregated demand level by increasing the stationary probabilities at levels between 100 and 400 for $M=2$ (Fig. 9(a)) and between 200 and 800 for $\mathrm{M}=4$ (Fig. 9(b)). This is in line with the observation in Fig. 6, where the most probable states are $\left\{3^{\prime}, 0\right\}$ and $\left\{0,3^{\prime}\right\}$. Therefore, the optimal decisions do not lead to the highest aggregated demand level but rather they aim to achieve a stable one to best manage resources. In contrast, the stationary probabilities for the Cautious and Greedy strategies are more spread out, which ultimately proves less favorable in terms of the system reward. In addition, as the WC increases, we still observe that the stationary probabilities increase and converge toward mid-range aggregate demand levels. Consequently, even at high WC levels, the best product portfolio strategy is apparently to smooth and reduce total aggregated demand. 


\subsubsection{Other portfolio compositions}

We further analyze the optimal decisions for a heterogenous portfolio composed of one functional and one innovative product. Fig. 10 shows the optimal decisions and the implied stationary probabilities. In Fig. 10(a) it can be seen that the optimal decisions are no longer symmetric (compared to Figs. 7(a) and 7(b)) and depict a complex decision structure. The optimal decisions benefit the innovative product over the functional product. This can readily
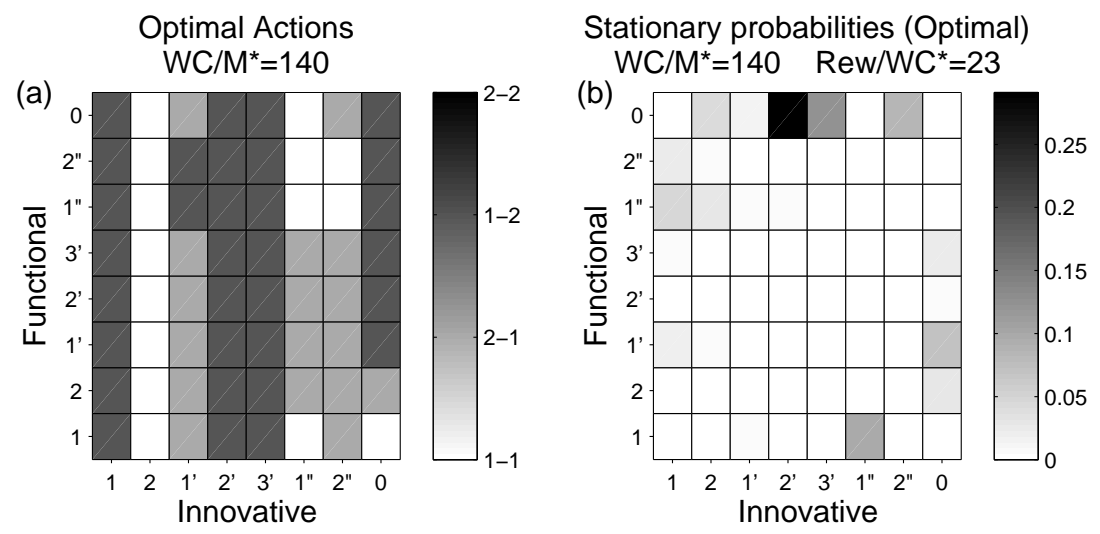

Figure 10: (a) Optimal decisions at the optimal WC level. (b) Stationary probabilities from the optimal decisions. (Heterogenous portfolio of a functional and an innovative product, $\mathrm{M}=2$ )

be seen in Fig. 10(b), in which the states with the highest likelihood are those where the innovative product is at its highest demand levels $\left\{3,2,2^{\prime}, 2^{\prime \prime}\right\}$ and the functional product is in state 0. Figs. 11(a) and 11(b) show that the Cautious and Greedy strategies result in a significantly lower average reward per WC than the optimal product portfolio strategy shown in Figs. 10(a) and 11(b).
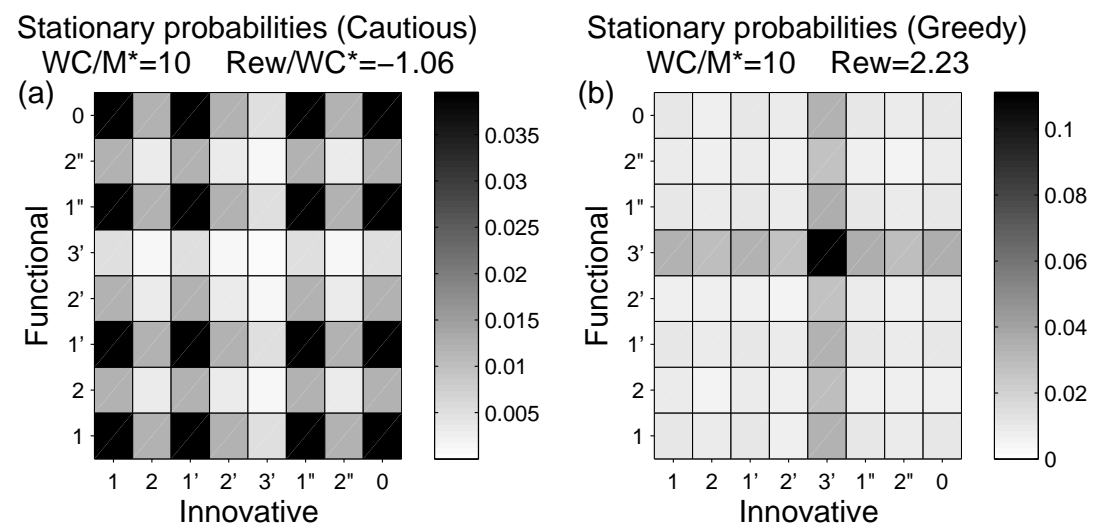

Figure 11: Stationary probabilities for the (a) Cautious and (b) Greedy strategies. (Heterogenous portfolio of a functional and an innovative product, $\mathrm{M}=2$ )

Since optimal decisions influence the average proportion of time that a product line is 
with or without products in the market (State 0), we analyze this measure for portfolios of two products. Table 2 contains the average proportion of time in the market for portfolios composed of (a) two innovative products (same as in Figs. 7 and 8), (b) one functional and one innovative product (same as in Figs. 10 and 11), and (c) two functional products. The proportions of time reveal that the optimal decisions often result in having only one product in the market, to achieve stable resource requirements. Moreover, these results show that the proportion of time in the market for each product line in the product portfolio depends heavily on the product characteristics (functional or innovative).

\begin{tabular}{l|cc} 
In & 0.42 & 0.16 \\
Out & 0.00 & 0.42 \\
\hline & Out & In
\end{tabular}

(a) Two innovative

\begin{tabular}{l|cc} 
In & 0.13 & 0.29 \\
Out & 0.00 & 0.57 \\
\hline & Out & In
\end{tabular}

(b) Functional \& Innovative

\begin{tabular}{l|cc} 
In & 0.41 & 0.18 \\
Out & 0.00 & 0.41 \\
\hline & Out & In
\end{tabular}

(c) Two functional

Table 2: Average proportion of time in or out of the market for two-product portfolios. $(\mathrm{M}=2)$

\subsection{Optimal product portfolio}

In this section, we use our MDP model to analyze the "ideal" product portfolio composition. If we can dynamically manage a product portfolio, Fig. 12 illustrates the best product portfolio for a company to carry with a given WC level. To build this graph, we compare the

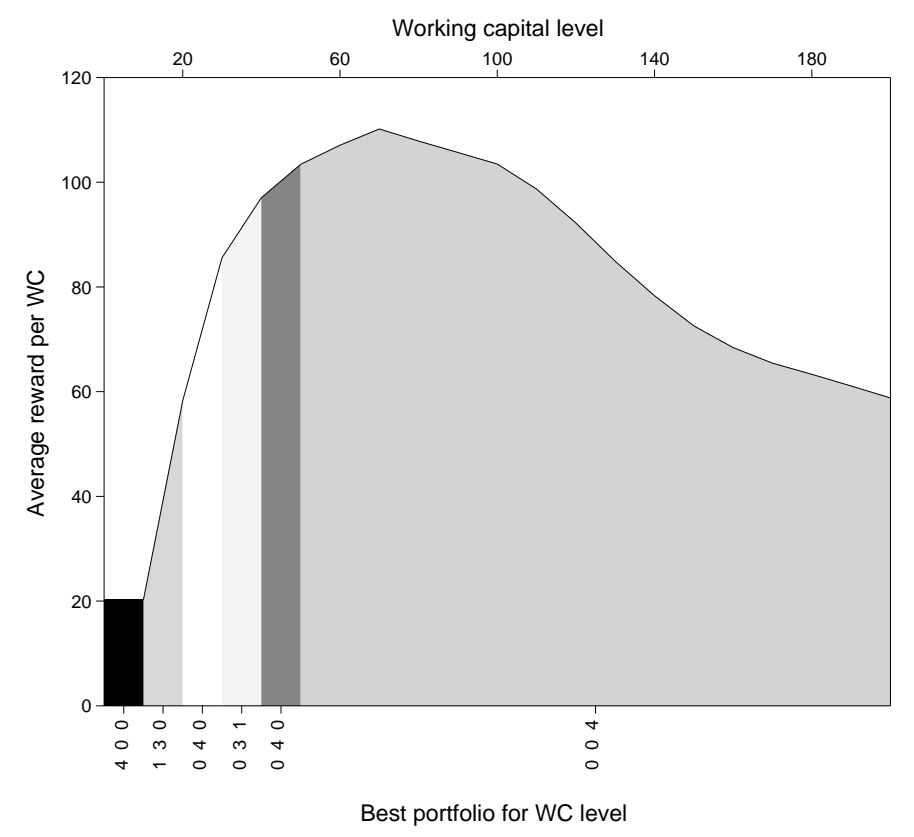

Figure 12: Portfolio composition with highest average reward per $\mathrm{WC}$ for different $\mathrm{WC}$ levels. $(\mathrm{M}=4)$

reward per WC for each possible combination of portfolios of four products comprising either 
of three product types: functional, innovative or average. The parameters of the "average" product type are right in the middle, between those of the functional and innovative product types (Section 4.1). The three numbers in the x-axis that runs from top to bottom provide the ideal number of innovative, average and functional products. From Fig. 12, we can clearly see how the best product portfolio varies depending on the level of available WC. With a WC of $K^{*}=70$, the company should carry four innovative products. For more restrictive WC levels, however, the ideal portfolio changes from more innovative to more functional. This trend strengthens as WC becomes more constraining, implying that in periods of lasting WC constraints, not only do the adequate marketing and launching decisions need to be made carefully, but also the portfolio composition ultimately needs to be revisited as proper support might no longer be given, which would prevent reaping the full benefits of launching an innovative product.

\section{Conclusion}

Companies have to constantly manage their portfolio of product lines. For increasingly more products, this involves adequately considering the product life cycle and its uncertainty. Therefore, portfolio composition, product launch timing, marketing, inventory levels, and the level of resources allocated all need to be coherently decided. In this paper, we develop a Markov decision process tool that includes all of these characteristics. The tractability and readability of the model stems from a constant WC assumption along with the access to shortterm debt. Yet, our model offers far greater insight into the complex decisions required to dynamically manage a product portfolio. To illustrate the usefulness of our tool, we perform analysis at three levels: 1) at the single product level to understand the effect of WC on rewards; 2) at the portfolio level, to understand the effects of jointly managing the portfolio and the required decisions to achieve its optimal behavior; 3) at the portfolio composition level, to understand how it may change under resource limitations. Besides providing a unified tool for portfolio management, our work provides the following managerial insights:

- Product portfolios benefit greatly from joint management, which reduces the amount of WC used per product and increases the benefits per allocated WC.

- The optimal decisions (regarding the launch timing, the marketing support and the WC level) are complex and dynamic, justifying the use of a comprehensive model, including 
the complex and multiple trade-offs involved. Cautious and Greedy policies lead to much lower profit.

- The optimal policy aims to reach a stable aggregate demand level and thus stable resource requirements, rather than temporarily reaching the highest aggregate demand level. Optimal decisions smooth not only aggregated demand levels but also cash flows and the length of time in and outside the market. This is important to ensure optimal use of resources and avoid high costs.

- Portfolio composition is a strategic decision that can also be made with the help of the proposed portfolio management tool. The optimal composition depends closely on the characteristics of admitted product types.

- Our results provide useful insights regarding the product rollover strategies of companies. We have shown that the optimal policy aims to smooth the resource requirements over time. This result is consistent with practical applications. For example, Apple has been following a multi-product roll strategy that aims to smooth aggregate demand levels and the resource requirements (Liao and Seifert, 2015; Kempster et al., 2014; Cross and Rogers, 2012). This strategy also allows Apple to first observe market demand, leading to a substantial resolution of demand uncertainty, and then minimize potential losses for the newer models (Biyalogorsky and Koenigsberg, 2014).

The type of insights presented highlight the value of a wide view in portfolio management and reveal that the decisions involved to take advantage of better joint management cannot be deduced from a simple analysis. Although we make an effort to keep our model realistic, we acknowledge that in order to reduce the number of states in our model, we have used simplifying assumptions to take advantage of the Markov property. The WC assumption and the access to short-term debt, for example, help to have renewal points at the end of each period. Moreover, different approximation of the ordering policies might be considered to better address product life cycle uncertainty. Nonetheless, we believe that these simplifications are not far from reality and have allowed for tractability and clarity in the analysis of all the interrelated features. Future work could study the effects of replenishment lead time since, most likely, it would exacerbate the effects of product life cycle uncertainty. Moreover, other 
aspects such as competition or market cycles may be included to paint an even more complete picture.

Appendix A Proof of the theorem

Without loss of generality, we consider two products jointly sold to the market. Let $x_{m}$ denote the demand and $Q_{m}$ the order quantity for product $m$, where $m \in\{1,2\}$. The mathematical model maximizing the total profit is

$$
\underset{\underset{\mathcal{H}_{j}, Q_{j}}{\operatorname{Maximize}} \quad z=p_{1} \mathcal{H}_{1}+p_{2} \mathcal{H}_{2}-c_{1} Q_{1}-c_{2} Q_{2}}{\text { subject to: }}
$$

$$
\begin{aligned}
& \mathcal{H}_{1}-Q_{1} \leq 0, \\
& \mathcal{H}_{1} \leq x_{1}, \\
& \mathcal{H}_{2}-Q_{2} \leq 0, \\
& \mathcal{H}_{2} \leq x_{2}, \\
& \mathcal{H}_{1}, \mathcal{H}_{2}, Q_{1}, Q_{2} \geq 0 .
\end{aligned}
$$

This model can be considered as a simpler version of Van Mieghem (1998). We now do not impose any working capital constraint. Therefore, this model can be considered as a special case in which the working capital constraint is not binding. Following the same procedure as in Van Mieghem (1998), we partition the demand space into four domains. Figure 13 illustrates the domains. The dual variables of our model are formalized for each domain as follows.

$$
\left(\alpha_{1}, \alpha_{2}, \beta_{1}, \beta_{2}\right)= \begin{cases}\left(0, p_{1}, 0, p_{2}\right) & \text { if } x \in \Omega_{1} \\ \left(0, p_{1}, p_{2}-c_{2}, 0\right) & \text { if } x \in \Omega_{2} \\ \left(p_{1}-c_{1}, 0, p_{2}-c_{2}, 0\right) & \text { if } x \in \Omega_{3} \\ \left(p_{1}-c_{1}, 0,0, p_{2}\right) & \text { if } x \in \Omega_{4}\end{cases}
$$




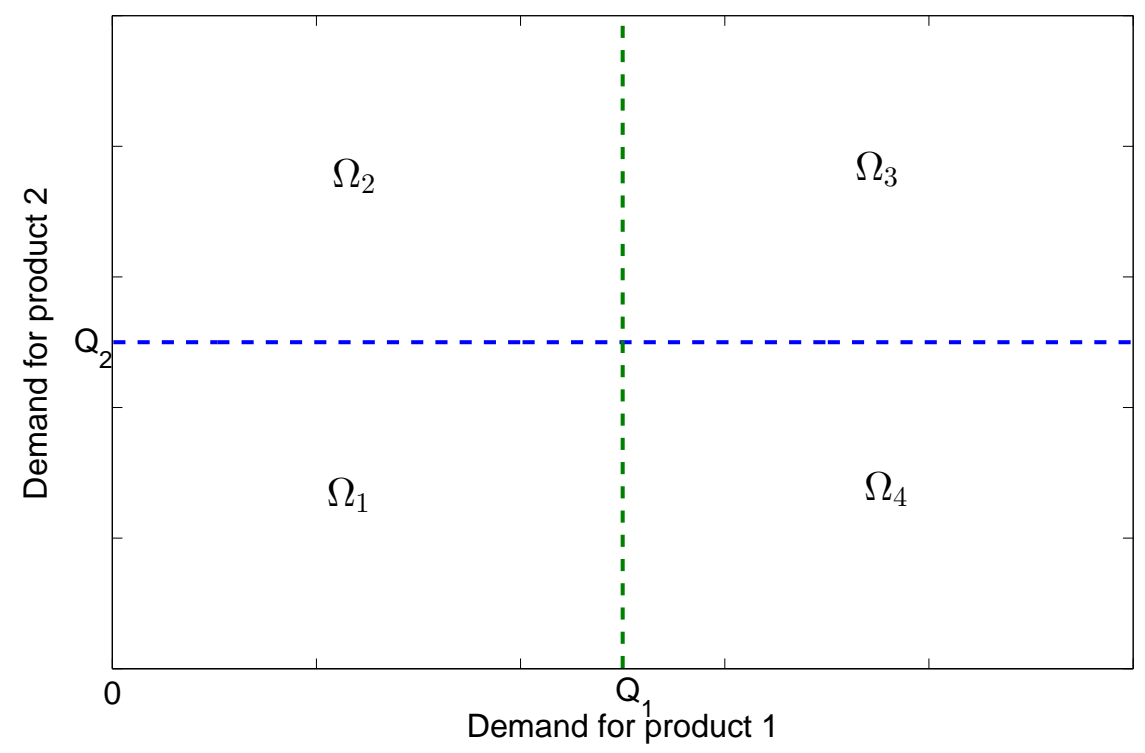

Figure 13: Parametric analysis of the linear programming model

Then, the optimal solution that comes from the dual problem satisfies the following conditions:

$$
\begin{aligned}
& \mathrm{E}\left(\alpha_{2}\right)=p_{1}-c_{1} \quad \Longrightarrow \quad F_{1}\left(Q_{1}\right)=\frac{p_{1}-c_{1}}{p_{1}} \\
& \mathrm{E}\left(\beta_{2}\right)=p_{2}-c_{2} \quad \Longrightarrow \quad F_{2}\left(Q_{2}\right)=\frac{p_{2}-c_{2}}{p_{2}}
\end{aligned}
$$

Therefore, the optimal solution reduces to the form of the newsvendor solution unless product pooling is taken into account. In our problem, it is not possible to pool inventory. Therefore, the optimal decision is made based on the marginal distribution of each product, and hence independent from the correlation between demand for the products. After calculating the optimal values for different products, we incorporate the working capital constraint by utilizing a simple allocation rule. We now add the working capital constraint to our mathematical model and analyze the optimal solution. The working capital constraint is written as follows.

$$
c_{1} Q_{1}+c_{2} Q_{2} \leq K .
$$


Then, the dual variables of our model are formalized for each domain as follows.

$$
\left(\alpha_{1}, \alpha_{2}, \beta_{1}, \beta_{2}, \lambda\right)= \begin{cases}\left(0, p_{1}, 0, p_{2}, \lambda_{1}\right) & \text { if } x \in \Omega_{1}, \\ \left(0, p_{1}, p_{2}-c_{2}, 0, \lambda_{2}\right) & \text { if } x \in \Omega_{2}, \\ \left(p_{1}-c_{1}, 0, p_{2}-c_{2}, 0, \lambda_{3}\right) & \text { if } x \in \Omega_{3}, \\ \left(p_{1}-c_{1}, 0,0, p_{2}, \lambda_{4}\right) & \text { if } x \in \Omega_{4},\end{cases}
$$

where

$$
\begin{aligned}
& \lambda_{1}=0, \quad \lambda_{2}=\frac{K_{2}}{K_{1}+K_{2}} \times \frac{p_{2}-c_{2}}{c_{2}} \\
& \lambda_{3}=\frac{K_{1}}{K_{1}+K_{2}} \times \frac{p_{1}-c_{1}}{c_{1}}+\frac{K_{2}}{K_{1}+K_{2}} \times \frac{p_{2}-c_{2}}{c_{2}}, \quad \lambda_{4}=\frac{K_{1}}{K_{1}+K_{2}} \times \frac{p_{1}-c_{1}}{c_{1}} .
\end{aligned}
$$

We note that the allocation policy, assuming that the working capital constraint is binding, determines the $\lambda$ in each domain. The optimality conditions are

$$
\begin{aligned}
& \mathrm{E}\left(\alpha_{2}+\lambda c_{1}\right)=p_{1}-c_{1}, \\
& \mathrm{E}\left(\beta_{2}+\lambda c_{2}\right)=p_{2}-c_{2} .
\end{aligned}
$$

Then, we get the following results:

$$
\begin{aligned}
& p_{1} F_{1}\left(Q_{1}\right)+\frac{K_{2}}{K_{1}+K_{2}} \times \frac{p_{2}-c_{2}}{c_{2}} c_{1}\left(1-F_{2}\left(Q_{2}\right)\right)+\frac{K_{1}}{K_{1}+K_{2}}\left(p_{1}-c_{1}\right)\left(1-F_{1}\left(Q_{1}\right)\right)=p_{1}-c_{1},(A) \\
& p_{2} F_{2}\left(Q_{2}\right)+\frac{K_{2}}{K_{1}+K_{2}}\left(p_{2}-c_{2}\right)\left(1-F_{2}\left(Q_{2}\right)\right)+\frac{K_{1}}{K_{1}+K_{2}} \times \frac{p_{1}-c_{1}}{c_{1}} c_{2}\left(1-F_{1}\left(Q_{1}\right)\right)=p_{2}-c_{2} .(
\end{aligned}
$$

The final equations imply that the optimal solution under working capital constraints only depends on the marginal distribution and is independent from the correlation between the demand for the products.

\section{References}

Ahiska, S. and King, R. (2010), 'Life cycle inventory policy characterizations for a singleproduct recoverable system', International Journal of Production Economics 124(1), 51-61.

Aitken, J., Childerhouse, P. and Towill, D. (2003), 'The impact of product life cycle on supply chain strategy', International Journal of Production Economics 85(2), 127-140. 
Anderson, C. (2009), The Longer Long Tail: How Endless Choice is Creating Unlimited Demand, Random House Business, New York.

Barksdale, H., Harris, C. et al. (1982), 'Portfolio analysis and the product life cycle', Long Range Planning 15(6), 74-83.

Biçer, I. and Seifert, R. W. (2015), Optimal production and investment policies under evolutionary demand risk. Working paper, Ecole Polytechnique Fédérale de Lausanne, Lausanne, Switzerland.

Biçer, I. (2015), 'Dual sourcing under heavy-tailed demand: an extreme value theory approach', International Journal of Production Research 53(16), 4979-4992.

Billington, C., Lee, H. and Tang, C. (1998), 'Successful strategies for product rollovers', Sloan Management Review 39(3), 23-30.

Biyalogorsky, E. and Koenigsberg, O. (2014), 'The design and introduction of product lines when consumer valuations are uncertain', Production and Operations Management 23(9), 1539-1548.

Chien, C., Chen, Y. and Peng, J. (2010), 'Manufacturing intelligence for semiconductor demand forecast based on technology diffusion and product life cycle', International Journal of Production Economics 128(2), 496-509.

Childerhouse, P., Aitken, J. and Towill, D. (2002), 'Analysis and design of focused demand chains', Journal of Operations Management 20(6), 675-689.

Choi, J., Cao, J., Romeijn, H., Geunes, J. and Bai, S. (2005), 'A stochastic multi-item inventory model with unequal replenishment intervals and limited warehouse capacity', IIE Transactions 37(12), 1129-1141.

Cooper, R., Edgett, S. and Kleinschmidt, E. (1999), 'New product portfolio management: practices and performance', Journal of Product Innovation Management 16(4), 333-351.

Cross, C. and Rogers, S. (2012), 'Apple product launches - are they becoming more frequent?', The Guardian . 
URL: $\quad$ http://www.theguardian.com/news/datablog/interactive/2012/oct/25/appleproduct-launches-timeline

Day, G. (1981), 'The product life cycle: analysis and applications issues', The Journal of Marketing 45(4), 60-67.

DeCroix, G. and Arreola-Risa, A. (1998), 'Optimal production and inventory policy for multiple products under resource constraints', Management Science 44(7), 950-961.

Dickinson, M., Thornton, A. and Graves, S. (2001), 'Technology portfolio management: optimizing interdependent projects over multiple time periods', Engineering Management, IEEE Transactions on 48(4), 518-527.

Ernst and Young (2010), 'All tied up', White Paper, Working Capital Management Report.

Fisher, M. (1997), 'What is the right supply chain for your product?', Harvard Business Review 75, 105-117.

Fox, G., Baker, N. and Bryant, J. (1984), 'Economic models for R\&D project selection in the presence of project interactions', Management Science 30(7), 890-902.

Gerhard, D., Brem, A., Baccarella, C. and Voigt, K. (2011), 'Innovation management and marketing in the high-tech sector: A content analysis of advertisements', International Journal of Management 28(1), 330-348.

Heidenberger, K., Schillinger, A. and Stummer, C. (2003), 'Budgeting for research and development: a dynamic financial simulation approach', Socio-Economic Planning Sciences $37(1), 15-27$.

Hess, J. and Lucas, M. (2004), 'Doing the right thing or doing the thing right: Allocating resources between marketing research and manufacturing', Management Science 50(4), 521526.

Hsueh, C. (2011), 'An inventory control model with consideration of remanufacturing and product life cycle', International Journal of Production Economics 133(2), 645-652.

Hu, G. and Bidanda, B. (2009), 'Modeling sustainable product lifecycle decision support systems', International Journal of Production Economics 122(1), 366-375. 
Hu, Q., Sobel, M. J. and Turcic, D. (2010), Optimization of inventory and dividends with risky debt. Working paper, Case Western Reserve University, Cleveland, OH.

Kavadias, S. and Chao, R. (2007), Resource allocation and new product development portfolio management, in 'Handbook of New Product Development Management', Elsevier/Butterworth-Heinemann, Oxford, UK, p. 135.

Kempster, J., Sparkes, M., Jones, S., Gunter, J., Williams, R., Moores, I. and Warman, M. (2014), 'Mac at 30 timeline: Apple's every major product', The Telegraph .

URL: $\quad$ http://www.telegraph.co.uk/technology/apple/10580156/Mac-at-30-timelineApples-every-major-product.html

Ketzenberg, M., Metters, R. and Semple, J. (2006), 'A heuristic for multi-item production with seasonal demand', IIE Transactions 38(3), 201-211.

Kleywegt, A. and Papastavrou, J. (1998), 'The dynamic and stochastic knapsack problem', Operations Research 46(1), 17-35.

Kleywegt, A. and Papastavrou, J. (2001), 'The dynamic and stochastic knapsack problem with random sized items', Operations Research 49(1), 26-41.

Li, H., Graves, S. and Rosenfield, D. (2010), 'Optimal planning quantities for product transition', Production and Operations Management 19(2), 142-155.

Li, L., Shubik, M. and Sobel, M. J. (2013), 'Control of dividends, capital subscriptions, and physical inventories', Management Science 59(5), 1107-1124.

Liao, S. and Seifert, R. W. (2015), 'On the optimal frequency of multiple generation product introductions', European Journal of Operational Research . DOI:10.1016/j.ejor.2015.03.041.

Lim, W. and Tang, C. (2006), 'Optimal product rollover strategies', European Journal of Operational Research 174(2), 905-922.

Littman, M. L., Dean, T. L. and Kaelbling, L. P. (1995), On the complexity of solving markov decision problems, in 'Proceedings of the Eleventh conference on Uncertainty in artificial intelligence', pp. 394-402. 
McGavin, E., Ward, J. and Schwarz, L. (1997), 'Balancing retailer inventories', Operations Research 45(6), 820-830.

Moon, I. and Silver, E. (2000), 'The multi-item newsvendor problem with a budget constraint and fixed ordering costs', Journal of the Operational Research Society 51(5), 602-608.

Nagarajan, M. and Rajagopalan, S. (2008), 'Inventory models for substitutable products: optimal policies and heuristics', Management Science 54(8), 1453-1466.

Protopappa-Sieke, M. and Seifert, R. (2010), 'Interrelating operational and financial performance measurements in inventory control', European Journal of Operational Research 204(3), 439-448.

Protopappa-Sieke, M. and Seifert, R. (2011), 'Interrelating operational and financial performance measurements in a multiproduct inventory system', International Journal of Services and Operations Management 10(3), 328-347.

Puterman, M. (1994), Markov decision processes: Discrete stochastic dynamic programming, John Wiley \& Sons, Inc. New York, NY, USA.

Schmidt, R. and Freeland, J. (1992), 'Recent progress in modeling R\&D project-selection processes', Engineering Management, IEEE Transactions on 39(2), 189-201.

Şen, A. (2008), 'The US fashion industry: A supply chain review', International Journal of Production Economics 114(2), 571-593.

Souder, W. (1973), 'Analytical effectiveness of mathematical models for R\&D project selection', Management Science 19(8), 907-923.

Van der Laan, E. and Salomon, M. (1997), 'Production planning and inventory control with remanufacturing and disposal', European Journal of Operational Research 102(2), 264-278.

Van Mieghem, J. A. (1998), 'Investment strategies for flexible resources', Management Science 44(8), 1071-1078.

Veinott Jr, A. (1965), 'Optimal policy for a multi-product, dynamic, nonstationary inventory problem', Management Science 12(3), 206-222. 
Wilhelm, W. and Xu, K. (2002), 'Prescribing product upgrades, prices and production levels over time in a stochastic environment', European Journal of Operational Research $138(3), 601-621$.

Zeballos, A. (2013), Inventory management with working capital restrictions, PhD thesis, Ecole Polytechnique Federale de Lausanne (EPFL), Switzerland. 